\title{
WAVE PROPAGATION OVER NON-UNIFORM ELECTRICAL CONDUCTORS*
} BY

\author{
M. I. PUPIN
}

\section{Introduction}

The main object of the mathematical theory developed in this paper is the analysis of the propagation of electrical waves over a conductor represented in Fig. $3, \S$ III, below. This conductor consists of a loop of wire $L_{1} \cdots L_{k+1}$ of length $2 l$ in which there are, at equidistant points, the so-called reactance points, a certain number of equal coils interposed dividing the loop into a number of equal parts, called the interstices of the loop. These coils may have condensers in series with them or they may have secondary circuits with condensers. The propagation of electrical waves over a periodically loaded loop of this kind is compared with that over a uniform loop having the same total inductance, resistance, and capacity. This uniform loop is called the corresponding uniform conductor of the periodically loaded loop.

A similar problem in mechanics is that of the forced and free vibrations of a periodically loaded heavy, flexible, inextensible string of finite length taking frictional resistances into account. To my knowledge, neither the electrical problem nor its corresponding mechanical problem has been investigated before.

It will be observed in the course of this paper that a study of the propagation of electrical waves over a periodically loaded conductor of this kind suggests forcibly an electromagnetic theory of emission and absorption of light by molecular complexes, which on account of the physical conception underlying it, if for no other reason, possesses many attractive features. In this theory the ether and the material ions imbedded in it correspond to the uniform wire and the reactance points considered in this paper. These matters, however, are of a more or less speculative character and have, therefore, no place here. But it should be noted that the physical problem discussed here was first suggested by speculations of this kind. It can be stated as follows: Under what conditions will the non-uniform conductor represented in Fig. 3 be approximately equivalent to its corresponding uniform conductor? Or, to be more precise : For what fre-

\footnotetext{
* Presented to the Society December 28, 1899. Received for publication January 23, 1900.
} 
quency will an electrical wave have approximately the same wave-length and the same damping or attenuation constant on one conductor as on the other?

The mathematical theory developed here gives a definite answer to this question. This answer can be stated in a few words and for that purpose it is desirable to introduce here a new technical term, the so.called angular distance of the interstices, that is, of the interval between consecutive reactance points. This interval is at any given frequency a definite fraction of the wave-length corresponding to that frequency. Let this fraction be $\phi / 2 \pi$; then $\phi$ is the angular distances of the interstices. The angular distance of a wave-length is, of course, $2 \pi$. The general rule expressing the conditions of equivalence of a non-uniform conductor to its corresponding uniform conductor can now be expressed as follows : For any given frequency a non-uniform conductor of the second type is equivalent to its corresponding uniform conductor as nearly as $\sin \phi / 2$ is to $\phi_{/} / 2$.

The higher the frequency the less resemblance will there be between a given non-uniform conductor and its corresponding uniform conductor. Conversely, if this resemblance is sufficiently close for a given frequency it will be closer for all lower frequencies. When the half wave-length under consideration becomes smaller than the interstices, then the resemblance, as far as that wave-length and all shorter wave-lengths are concerned, ceases altogether.

A brief summary of this paper will now be given.

The main object of this research is the solution of the problem of $\S$ III. This solution depends on the solution of the problem of $\S$ II, and this again is moulded after the pattern of the solution of the problem of $\S \mathrm{I}$.

In $\S I$ the wave propagation over a uniform wire conductor represented in Fig. 1 is discussed. The effect of the transmitting apparatus $A$ and of the receiving apparatus $B$ is taken into account. Equation (5) is the most general solution of this case. The propagation of waves of both forced and free periods is easily deduced from it. This particular form of the general solution is new and it was selected because it is best suited for comparing the propagation of waves over the non-uniform conductors of $\S \S$ II and III with that over their corresponding uniform conductors.

In $\S$ II the wave propagation over a non-uniform conductor of the first type represented in Fig. 2 is discussed. This conductor consists of a certain number of equal coils $L_{1}, \cdots, L_{n}$ which are connected in series. A certain number of equal condensers, one at each juncture between two consecutive coils, connect this conductor to ground. A transmitting apparatus $A$ and a receiving apparatus $B$ are present. The general solution of this problem, equation $(6 a)$, is moulded after the pattern of equation (5) of $\S \mathrm{I}$. Both forced and free oscillations on a conductor of this kind are considered, and the conditions under which it becomes equivalent to its corresponding uniform conductor are worked out at considerable length. The problem of this section and its solution are both new. 
The problem of $\S$ III can, from a purely mathematical point of view, be stated as follows: Find the integral of the following partial differential equation :

$$
L \frac{d^{2} y}{d t^{2}}+R \frac{d y}{d t}=\frac{1 \partial^{2} y}{C} \frac{\partial s^{2}}{2}
$$

and determine it in such a way as to satisfy $k+2$ boundary conditions. Determine also the conditions under which this integral will be equivalent to the integral represented in equation (5), $§ \mathrm{I}$.

Equation (2) represents the most general solution, and the constants $x_{1}, x_{2}, \cdots, x_{k+2}$ have to be determined from the $k+2$ boundary conditions. The principal mathematical difficulty here reduces itself then to the proper mathematical formulation of these boundary conditions so as to obtain a system of equations which can be readily solved. Such a system is system (3) of this section. It is of the same form as system (6) of $\S$ II, the solution of which was obtained in that section. Equations (4) and (5) are thus obtained. Equation (5) is the most general solution, and when this equation becomes very nearly the same as equation (5) of $\S I$ then the non-uniform conductor of this section becomes equivalent to its corresponding uniform conductor. This equivalence cannot be decided without a careful study of the wave-lengths and of the damping constants of waves of different periods. This study is recorded in the remaining portion of this section. This section is also entirely new.

The work given in $\S I$ and a portion of that given in $\S$ II, together with a description of experimental investigations bearing upon the same, were published in vol. XVI of the Transactions of the American. Institute of Electrical Engineers for 1899. Additional experimental investigations bearing upon the principal problem, that is the problem of $\S$ III, will be published in the near future.

\section{§ I. Wave Propagation along a uniform linear Conductor.}

\section{A. Waves of forced period.}

The conductor is a loop of wire $A B$ (Fig. 1). At one point of the loop is a transmitting apparatus $A$, at the opposite point is a receiving apparatus $B$. The distance between $A$ and $B$ is $l$, equal to one-half the length of the whole loop. The distance of any element $d s$ from $A$ is $s$.

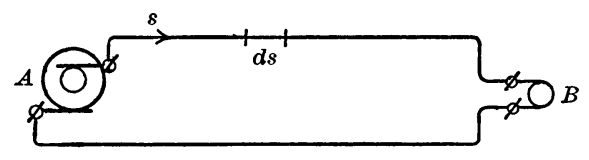

FrG. 1 
Let $L, R$, and $C$, denote the inductanee, resistance, and capacity, respectively, per unit length. Let $y$ be the current at any point $s$. Then

$$
L \frac{d^{2} y}{d t^{2}}+R \frac{d y}{d t}=\frac{1 \partial^{2} y}{C} \frac{\partial s^{2}}{d}
$$

is the well known equation of propagation.

To formulate the two boundary conditions, one at the receiving and the other at the transmitting apparatus, let $L_{0}, R_{0}, C_{0}$, and $L_{1}, R_{1}, C_{1}$, respectively be the inductance, resistance, capacity, of the transmitting and of the receiving apparatus. Each contains a condenser in series with its inductance and resistance. Let the e. m. f. impressed by $A$ be of type $E e^{i p t}$. The boundary equations can now be expressed as follows :

where

$$
\left\{\begin{array}{lr}
-2\left(\frac{\partial y}{\partial s}\right)_{s=0}=\left(D_{0}-h_{0} y\right)_{s=0} & \text { at transmitting apparatus } \\
+2\left(\frac{\partial y}{\partial s}\right)_{s=l}=-\left(h_{1} y\right)_{s=l} & \text { at receiving apparatus }
\end{array}\right.
$$

$$
\begin{array}{cc}
D_{0}=i p C_{0} E e^{i p t}, \\
h_{0}=C_{0}\left(-p^{2} \lambda_{0}+i p R_{0}\right), & h_{1}=C_{1}\left(-p^{2} \lambda_{1}+i p R_{1}\right), \\
\lambda_{0}=L_{0}-\frac{1}{p^{2} C_{0}}, & \lambda_{1}=L_{1}-\frac{1}{p^{2} C_{1}} .
\end{array}
$$

The physical character of the problem suggests the following solution :

$$
y=K_{1} \cos \mu \xi+K_{2} \sin \mu \xi,
$$

where $\xi=l-s$, and $K_{1}$ and $K_{2}$ are proportional to $e^{i p t}$.

Equation (1) is satisfied for all values of $K_{1}$ and $K_{2}$ if

From this we get

$$
C\left(-p^{2} L+i p R\right)=-\mu^{2}=-(a+i \beta)^{2} .
$$

$$
\begin{aligned}
& a=\sqrt{\frac{1}{2} p C\left\{\sqrt{p^{2} L^{2}+R^{2}+p L}\right\}}, \\
& \beta=\sqrt{\frac{1}{2} p C\left\{\sqrt{p^{2} L^{2}+R^{2}}-p L\right\}} .
\end{aligned}
$$

When $p L$ is large in comparison with $R$,

$$
a=p \sqrt{L C}, \quad \beta=\frac{R}{2} \sqrt{\bar{L}} *
$$

\footnotetext{
* Observe that in the case of a twin conductor where the mutual capacity is large in comparison with the capacity of each conductor to earth we must use $2 R$ and $2 L$ in place of $R$ and $L$.
} 
The boundary equations will be satisfied if

where

$$
K_{1}=\frac{2 \mu D_{0}}{F^{-}}, \quad K_{2}=\frac{h_{1} D_{0}}{F^{\prime}}
$$

$$
F=\left(h_{0} h_{1}-4 \mu^{2}\right) \sin \mu l+2 \mu\left(h_{0}+h_{1}\right) \cos \mu l .
$$

Hence (3) can now be written :

$$
y=\left(2 \mu \cos \mu \xi+h_{1} \sin \mu \xi\right)_{F}^{D_{0}}
$$

When $h_{0}=h_{1}=0$,

$$
y=-\frac{D_{0} \cos \mu \xi}{2 \mu \sin \mu l}
$$

Equation (5) is a complete solution for the propagation of waves of forced period. It represents simple harmonic damped waves. The most essential elements which enter into the description of such waves are the wave-length $\lambda$ and the damping or attenuation factor. These can easily be calculated. Since $\mu=a+i \beta$, we shall have $\lambda=2 \pi / a$; the attenuation factor is $e^{-\beta \xi}$.

It is evident that $\beta$ diminishes as $L$ increases. A high reactance per unit length means small attenuation and a slow speed of propagation.

\section{B. Waves with a natural period.}

Free oscillations are readily calculated for a few special cases. Equation (5) is a general solution for free oscillations also, provided, however, that $\mu$ has such a value as to make $F=0$, since $D_{0}=0$; that is, we must have

$$
\left(h_{0} h_{1}-4 \mu^{2}\right) \sin \mu l+2 \mu\left(h_{0}+h_{1}\right) \cos \mu l=0 ;
$$

but, of course, in this case,

$$
h_{0}=k C\left(k \lambda_{0}+R_{0}\right), \quad h_{1}=k C\left(k \lambda_{1}+R_{1}\right), \quad-\mu^{2}=k C(k L+R) .
$$

Equation (6) is a transcendental equation and can be readily solved in a few simple cases.

Case 1.-The transmitting and receiving apparatus are not present.

In this case $h_{0}=h_{1}=0$. Equation (6) reduces to

Therefore

$$
\sin \mu l=0 \text {. }
$$

$$
\mu=s \pi / l,
$$

where $s$ can have any integral value from 1 to $\infty$. The periods of free oscillation are calculated from the equation : 
Therefore

$$
-\mu^{2}=k^{2} L C+k R C=-\frac{s^{2} \pi^{2}}{l^{2}} .
$$

$$
k=-\frac{R}{2 L} \pm \sqrt{-1} \sqrt{\frac{1 s^{2} \pi^{2}}{L C} \frac{R^{2}}{4 l^{2}}}=-\frac{R}{2 L} \pm i k_{s} .
$$

There is, therefore, an infinite number of periods which are harmonically related to each other unless the damping constant $R / 2 L$ is not negligibly small in comparison with $\pi^{2} / l^{2} L C$.

The most general solution of this case can be written :

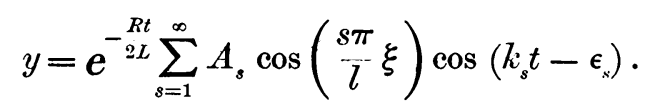

The wave-lengths are $2 l / 1,2 l / 2,2 l / 3, \cdots, 2 l_{j} s, \cdots$.

Case 2.-The transmitting apparatus is not present and in place of the receiving apparatus there is a break in the wire.

In this case $h_{0}=0, h_{1}=\infty$. Equation (6) reduces to

Therefore

$$
\cos \mu l=0 \text {. }
$$

$$
\begin{gathered}
\mu=\frac{2 s+1 \pi}{l} \overline{2} \\
k=-\frac{R}{2 L} \pm i \sqrt{\frac{1}{L C}\left(\frac{2 s+1 \pi}{l} \frac{\pi}{2}\right)^{2}-\frac{R^{2}}{4 L^{2}}}=-\frac{R}{2 L}+i k_{2 s+1}, \\
y=e^{-\frac{R}{2 L} t} \sum_{s=0}^{\infty} A_{2 s+1} \sin \left(\frac{2 s+1}{l} \frac{\pi}{2} \xi\right) \cos \left(k_{2 s+1}-\epsilon_{2 s+1}\right) .
\end{gathered}
$$

The wave-lengths are $4 l / 1,4 l / 3,4 l / 5, \ldots$.

The damping factor is in both cases the same for all frequencies; hence the color of the complex harmonic vibration remains unchanged during the whole epoch while the vibrations last.

\section{§ II. Electrical Oscillations on a non-uniform Conductor of THE FIRST TYPE.}

The conductor consists of $2 n$ equal coils, $L_{1}, L_{2}, \ldots, L_{n}$ (Fig. 2) connected in series so as to form a closed loop. At one point $A$ of this loop is an alternator, at the opposite point is a receiving apparatus $B$. At equal distances $2(n-1)$ equal condensers, $C_{1}, \ldots, C_{n-1}$, connect the conductor to ground. The whole loop 
is thus divided into $2 n-2$ component circuits, $1,2, \ldots, 2 n-2$. It is evident that in the limit when $n$ becomes infinitely large, this conductor becomes an ordinary telegraph or telephone line with uniformly distributed resistance, capacity, and inductance. The question now arises: Under what conditions will a conductor of this kind become equivalent, with sufficient approximation, to a uniform line, even when $n$ is not infinitely large? This problem does not seem to have been solved before. In its main features it is similar to that which Lagrange solved in his Mécanique Analytique, pt. II, sec. VI, the problem, namely, of the free vibrations of a weightless string, !fixed at its two

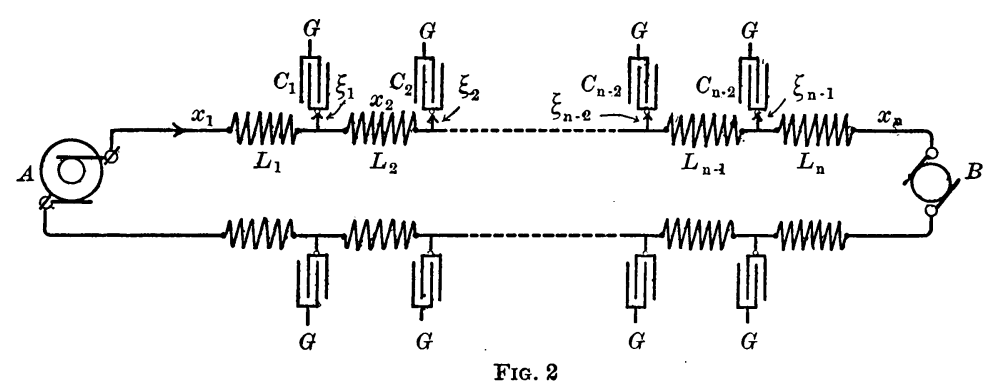

ends, and loaded at equidistant points by equal weights. But it is much more general because frictional resistances are taken into consideration, and also because forced as well as free oscillations are considered.

Let $L_{0}$ and $L_{1}, R_{0}$ and $R_{1}, C_{0}$ and $C_{1}$ be the inductance, ohmic resistance, capacity of $A$ and $B$, respectively. Let $L, R, C$ be the corresponding quantities of the coils and condensers in the several component circuits.

The real part of $E e^{i j t}$ is the e. m. f. impressed by alternator $A$.

Let $x_{1}, \ldots, x_{n}$ be the currents in the component circuits $P_{1}, \ldots, P_{n-1}$ the differences of potential in the line condensers, $C_{1}, C_{2}, \ldots, C_{n-1} ; P_{0}$ and $P^{\prime}$ the differences of potential in the condensers in $A$ and $B$ respectively; and $\xi_{1}, \cdots, \xi_{n-1}$ the condenser currents.

We shall have

$$
\left\{\begin{array}{l}
\xi_{1}=C \frac{d P_{1}}{d t}, \quad \xi_{2}=C \frac{d P_{2}}{d t}, \cdots \\
\xi_{1}=x_{1}-x_{2}, \quad \xi_{2}=x_{2}-x_{3}, \cdots
\end{array}\right.
$$

\section{A. Forced oscillations.}

Stating the law of equality of action and reaction for each component circuit, we obtain the following $n$ differential equations : 


$$
\left\{\begin{array}{c}
\left(L_{0}+2 L\right) \frac{d x_{1}}{d t}+\left(R_{0}+2 R\right) x_{1}+2 P_{1}+P_{0}=E e^{i p t} \\
L \frac{d x_{2}}{d t}+R x_{2}+P_{2}-P_{1}=0 \\
\cdot \cdot \cdot \cdot \cdot \cdot \cdot \cdot \\
L \frac{d x_{n-1}}{d t}+R x_{n-1}+P_{n-1}-P_{n-2}=0 \\
\left(L_{1}+2 L\right) \frac{d x_{n}}{d t}+\left(R_{1}+2 R\right) x_{n}-2 P_{n-1}^{-}+P^{\prime}=0
\end{array}\right.
$$

When the steady state has been reached, the currents will be, just like the impressed e. m. f., simple harmonics of the time $t$, that is,

$$
x_{1}=A_{1} e^{i p t}, \quad x_{2}=A_{2} e^{i p t}, \cdots,
$$

where $A_{1}, A_{2}, \cdots$ are complex quantities.

Differentiating each member of (2) and substituting from (3) and (1) we obtain :

where

$$
\left\{\begin{array}{c}
h x_{1}+\xi_{1}-0=D \\
h x_{2}+\xi_{2}-\xi_{1}=0 \\
\cdot \cdot \cdot \cdot \\
h x_{n-1}+\xi_{n-1}-\xi_{n-2}=0 \\
h x_{n}+0-\xi_{n-1}=-h_{1} x_{n}
\end{array}\right.
$$

$$
h=C\left(-p^{2} L+i p R\right), \quad h_{1}=\frac{1}{2} C\left(-p^{2} \lambda_{1}+i p R_{1}\right),
$$

$$
\begin{gathered}
D=\frac{1}{2} i p C E e^{i p t}-\frac{1}{2} C\left(-p^{2} \lambda_{0}+i p R_{0}\right)=D_{0}-h_{0} x_{1} \\
\lambda_{0}=L_{0}-\frac{1}{p^{2} C_{0}^{\prime}}, \quad \lambda_{1}=L_{1}-\frac{1}{p^{2} C_{1}^{\prime}} .
\end{gathered}
$$

Another form is obtained by substituting for $\xi_{1}, \xi_{2}, \cdots$ as follows :

(6)

$$
\left\{\begin{array}{l}
(h+1) x_{1}+0-x_{2}=D=D_{0}-h_{0} x_{1} \\
(h+2) x_{2}-x_{1}-x_{3}=0 \\
\cdot \cdot \cdot \cdot \cdot \\
(h+2) x_{n-1}-x_{n-2}-x_{n}=0 \\
(h+1) x_{n}-x_{n-1}-0=-h_{1} x_{n}
\end{array}\right.
$$


Equation (3) of § I suggests the following solution :

$$
x_{m}=K_{1} \cos 2(n-m) \theta+K_{2} \sin 2(n-m) \theta .
$$

If $h+2=2 \cos 2 \theta$, then all the equations except the first and the last will be satisfied for all values of $K_{1}$ and $K_{2}$. These two equations, which correspond to the boundary equations of $\S \mathrm{I}$, will be also satisfied if we assign to $K_{1}$ and $K_{2}$ suitable values, as follows :

$$
\begin{gathered}
K_{2}=\frac{\left(h_{1}-1\right)+\cos 2 \theta}{\sin 2 \theta} K_{1}, \\
K_{1}=\frac{D_{0} \sin 2 \theta}{h_{0} h_{1} \sin 2(n-1) \theta-4 \sin ^{2} \theta} \frac{\sin 2 n \theta+2\left(h_{0}+h_{1}\right) \sin \theta \cos (2 n-1) \theta}{} .
\end{gathered}
$$

We can now write

$$
\text { (7) } x_{n}=\frac{\left[2 \sin \theta \cos (2 n-2 m+1) \theta+h_{1} \sin 2(n-m) \theta\right] D_{0}}{h_{0} h_{1} \sin 2(n-1) \theta-4 \sin ^{2} \theta \sin 2 n \theta+2\left(h_{0}+h_{1}\right) \sin \theta \cos (2 n-1) \theta} \text {. }
$$

As $\theta$ is a complex angle, the forced oscillations of type $e^{i p t}$ on a non-uniform conductor of this kind are simple harmonic damped oscillations. The similarity between this conductor and a uniform wire will be discussed presently.

When $h_{0}=h_{1}=0$ we obtain

$$
x_{m}=-\frac{D_{0} \cos (2 n-2 m+1) \theta}{2 \sin \theta \sin 2 n \theta} .
$$

\section{B. Free oscillations.}

Equation (7) holds for free as well as for forced oscillations. But since $D_{0}=0$ in the case of free oscillations, it follows that the denominator of (7) must vanish to prevent the vanishing of all currents. We shall therefore have

$$
h_{0} h_{1} \sin (2 n-2) \theta-4 \sin ^{2} \theta \sin 2 n \theta+2\left(h_{0}+h_{1}\right) \sin \theta \cos (2 n-1) \theta=0 .
$$

From this equation, $\theta$ and the corresponding periods and damping constants have to be determined. A solution can be readily obtained for a small number of cases; the two most important will be considered here.

First case. The transmitting and the receiving apparatus are not present. In this case $h_{0}=h_{1}=0$, and

$$
x_{m}=B \cos (2 n-2 m+1) \theta .
$$

It is found from (8) that (9) is actually the solution of the differential equations (6) for $h_{0}=h_{1}=D_{0}=0$, provided that 


$$
\theta=\frac{s \pi}{2 n},
$$

where $s$ is any integer from 1 to $2 n$.

Hence the most general solution will be :

$$
x_{m}=\sum_{s=1}^{2 n} B_{s} \cos (2 n-2 m+1) \frac{s \pi}{2 n} .
$$

But it should be observed now that $x_{m}$ is a periodic function of the time, that is,

$$
x_{m}=\sum K_{s} e^{p^{t}} \text {. }
$$

The constant $p_{s}$, which measures the period and the damping constant of the free oscillation, is determined from the relation :

$$
h=-4 \sin ^{2} \theta \text {. }
$$

In the case of free oscillations,

Hence

$$
h=p^{2} L C+p R C, \quad \theta=\frac{s \pi}{2 n},
$$

$$
p_{s}^{2} L C+p_{s} R C=-4 \sin ^{2} \frac{s \pi}{2 n} \text {. }
$$

Before solving this equation, it is desirable to make the following substitutions :-

Let $L^{\prime}, C^{\prime}$, and $R^{\prime}$ be the total inductance, capacity, and resistance, respectively, of one-half of the conductor, then

$$
L=\frac{L^{\prime}}{n}, \quad C=\frac{C^{\prime}}{n}, \quad R=\frac{R^{\prime}}{n}
$$

Let $l$ denote the half length of a uniform wire having $\sigma, r$, and $c$, for inductance, resistance, and capacity per unit length, and let it have the same total inductance, resistance, and capacity as the non-uniform conductor. Since

we shall have

$$
l \sigma=L^{\prime}, \quad l r=R^{\prime}, \quad l c=C^{\prime}
$$

$$
L=\frac{l \sigma}{n}, \quad C=\frac{l c}{n}, \quad R=\frac{l r}{n} .
$$

This uniform wire having the same total inductance, resistance, and capacity as the non-uniform conductor, will be called the corresponding uniform conductor.

From (11) we obtain

$$
\frac{l^{2}}{n^{2}}\left(p_{s}^{2} \sigma c+p_{s} c r\right)=-4 \sin ^{2} \frac{s \pi}{2 n},
$$


whence

$$
p_{s}=-\underset{2 \lambda}{r} \pm \sqrt{-1} \sqrt{\frac{1}{\sigma c} \frac{4 n^{2}}{l^{2}} \sin ^{2} \frac{s \pi}{2 n}-\frac{r^{2}}{4 \lambda^{2}}}=-\frac{r}{2 \lambda} \pm i k_{s}
$$

Equation (10) now becomes :

$$
x_{m}=e^{-\frac{r t}{2 \sigma}} \sum_{s=1}^{2 n} A_{s} \cos (2 n-2 m+1) \frac{s \pi}{2 n} \cos \left(k_{s} t-\epsilon_{s}\right) .
$$

It is clear that the oscillations on the non-uniform conductor of the first type have the same damping constant as the oscillations on the corresponding uniform conductor. It will be shown presently that under certain conditions they will also have the longer periods, up to a certain limit, very nearly the same as the corresponding uniform conductor. For these periods, then, the non-uniform conductor will be equivalent to its corresponding uniform conductor.

Second case. The transmitting apparatus is not present, and in place of the receiving apparatus there is a break in the line at $B$.

In this case $h_{0}=0, h_{1}=\infty$. Equation (9) gives :

provided that

$$
x_{m}=B \sin (2 n-m+2) \theta \text {, }
$$

or

$$
\cos (2 n-1) \theta=0,
$$

We shall have, therefore,

$$
\theta=\frac{2 s+1 \pi}{2 n-12}
$$

Hence

$$
p_{2 s+1}=-\frac{r}{2 \sigma} \pm i \sqrt{\frac{1}{\lambda c} \frac{4 n^{2}}{l^{2}} \sin ^{2} \frac{2 s+1 \pi}{2 n-12}-\frac{r^{2}}{4 \lambda^{2}}}=-\frac{r^{r}}{2 \sigma} \pm i k_{2 s+1} .
$$

$$
x_{m}=e^{-\frac{r t}{2 \sigma}} \sum_{s=0}^{2 n} A_{2 s+1} \sin (2 n-2 m+2) \frac{2 s+1 \pi}{2 n-12} \cos \left(k_{2 s+1} t-\epsilon_{2 s+1}\right) .
$$

The remark in the preceding case regarding the damping constant and the free periods also applies here.

The wave-lengths of free oscillations. - The angles $s \pi / 2 n$ and $(2 s+1) \pi / 2(2 n-1)$ have an interesting physical meaning which will be brought out by considering the wave-lengths of the oscillations. Consider one of the component harmonics of $x_{m}$ in Case I, say

$$
\xi_{m, s}=A_{s} \cos (2 n-2 m+1) \frac{s \pi}{2 n} \cos \left(k_{s} t-\epsilon_{s}\right) \text {. }
$$

Compare it with the corresponding component of $x_{m_{1}}$, that is, with

$$
\xi_{m_{1}, s}=A_{s} \cos \left(2 n-2 m_{1}+1\right) \frac{s \pi}{2 n} \cos \left(k_{s} t-\epsilon_{s}\right) .
$$


If they are a wave-length apart, $\xi_{m, s}=\xi_{m_{1}, s}$ and $m_{1}-m$ is equal to the number of coils covered by one wave-length. But in this case,

therefore

$$
(2 n-2 m+1) \frac{s \pi}{2 n}=\left(2 n-2 m_{1}\right) \frac{s \pi}{2 n}+2 \pi ;
$$

$$
m_{1}-m=\frac{2 n}{s}=\nu_{s} \text {. }
$$

Hence $\nu_{s}$ is the number of coils covered by a wave-length which corresponds to the harmonic $s$. It can be shown that, in the second case, $2(2 n-1) / 2 s+1=\nu_{s}$. For $s \pi / 2 n$ and $(2 s+1) \pi / 2(2 n-1)$ we can, therefore, write $\pi / \nu_{s}=2 \pi / 2 \nu_{s}$. The physical meaning of $2 \pi / \nu_{s}$ can now be readily fixed. A coil represents a definite fraction of a wave-length, and this fraction will have a different value for different harmonics. The higher the harmonic the shorter will be the wavelength and therefore, the larger will be the value of this fraction. It is convenient, however, to measure this fraction in terms of an angle instead of in terms of a wave-length. If we arbitrarily assume that an angular distance $2 \pi$ corresponds to a wave-length then an angular distance $2 \pi / n$ will correspond to the $n$th part of a wave-length. With this understanding,

$$
s \pi / 2 n \text { and }(2 s+1) \pi / 2(2 n-1),
$$

that is $2 \pi_{i}^{\prime} 2 \nu_{s}$ represents one half of the angular distance covered by a coil.

\section{A non-uniform conductor of the first type compared with its corresponding uniform wire.}

a. Similarity with respect to free oscillations.-Comparing the expressions for the free periods of oscillation which were obtained in sections I and II we see that as long as $\pi / \nu_{s}$ can be written for $\sin \pi / \nu_{s}$, so long will the periods of free oscillations of the non-uniform conductor be nearly the same as those of its corresponding uniform conductor. We have therefore the simple rule : $A$ nonuniform conductor of the first type represents its corresponding uniform conductor as nearly as one half of the angular distance covered by one of its coils represents the sine of that distance. The non-uniform conductor employed by me* in my experiments had 400 coils. In this case

$$
\frac{\pi}{\nu_{s}}=\stackrel{s \pi}{400} .
$$

For $s=25$, we have $\pi / \nu_{s}=\pi / 16$. Now $\sin \pi / 16$ differs from $\pi / 16$ by $\frac{2}{3}$ of one per cent of the value of $\pi / 16$. Hence the period of the 25 th harmonic of my non-

\footnotetext{
* See paper cited in the introduction.
} 
uniform conductor differs from that of the same harmonic on the corresponding uniform wire by less than $\frac{2}{3}$ of one per cent. For lower harmonics the difference is smaller. The 25 th harmonic had approximately 3,500 p. p. s. Hence up to the 25 th harmonic, that is up to 3,500 p. p. s., the non-uniform conductor employed by me had nearly the same free periods as its corresponding uniform conductor.

b. Similarity with respect to forced oscillations.-The wave-length and the attenuation constant corresponding to a given frequency speed $p_{s}$ can be studied by studying the angle $\theta$ from the following equation :

or

$$
\begin{aligned}
-4 \sin ^{2} \theta_{s}=-p_{s}^{2} L C+i p_{s} R C=\frac{l^{2}}{n^{2}}\left[-p_{s}^{2} \rho C+i p_{s} r C\right] \\
=-\frac{l^{2}}{n^{2}} \mu_{s}^{2}=-\frac{l^{2}}{n^{2}}\left(a_{s}+i \beta_{s}\right)^{2},
\end{aligned}
$$

$$
\sin \theta_{s}=\frac{1}{2} \frac{l}{n}\left(a_{s}+i \beta_{s}\right)
$$

where $\rho, C$, and $r$, are the inductance, capacity, and resistance, respectively, per unit length of the corresponding uniform conductor; and $\mu_{s}, a_{s}$, and $\beta_{s}$ have the same meanings as in $\S \mathrm{I}$.

Referring now to equation (4), $\$$ I, we shall find that

$$
a_{s} \geqq \beta_{s} \text {. }
$$

If, therefore, $l a_{s} / 2 n$ is sufficiently small we can put

$$
\theta_{s}=\frac{1}{2} \frac{l}{n}\left(a_{s}+i \beta_{s}\right)=\frac{1}{2} \frac{l}{n} \mu_{s} \text {. }
$$

When this substitution is made in (7) and $(7 a)$ these equations will transform into $(5)$ and $(5 a)$ of $\S \mathrm{I}$, which shows that under these conditions the non-uniform conductor of the first type becomes approximately equivalent to its corresponding uniform conductor, the degree of approximation being the same as that of $\sin \theta_{s}$ to $\theta_{s}$.

The physical meaning of this can readily be made ciear. If by $\lambda_{s}$ we denote the wave-length on the corresponding uniform conductor, corresponding to the frequency speed $p_{s}$, then according to $\S \mathrm{I}$,

$$
a_{s}=\frac{2 \pi}{\lambda_{s}}, \quad \frac{l}{n} a_{s}=\frac{l}{n} \frac{2 \pi}{\lambda_{s}} .
$$

Let $n_{s}$ be the number of coils on the non-uniform conductor which cover a wave-length of frequency speed $p_{s}$, and let $\phi_{s}$ be the angular distance covered by a coil, then

$$
\phi_{s}=\frac{2 \pi}{n_{s}} .
$$


Again

or

Hence

$$
n: n_{s}:: l: \lambda_{s}
$$

$$
n: \frac{2 \pi}{\phi_{s}}:: l: \lambda_{s} \text {. }
$$

$$
\frac{l 2 \pi}{n \lambda_{s}}=\frac{l}{n} a_{s}=\phi_{s}
$$

The substitution mentioned above will therefore be permissible when $\frac{1}{2} \phi_{s}$ (that is, one-half of the angular distance covered by a coil) is approximately equal to $\sin \phi_{s} / 2$. We have here the same rule as in the case of free oscillations. It will be shown in $\S$ III that this rule is also applicable to non-uniform conductors of the second type, for waves of both forced and free periods.

\section{§III. Wave Propagation along a non-uniform Conductor of the SECOND TYPE.}

\section{A. Waves of forced periods.}

The conductor is represented in the diagram of Fig. 3. A long uniform wire forms a loop $L_{1} \cdots L_{k+2}$. At equal intervals are inserted in series $2 k+2$ equal

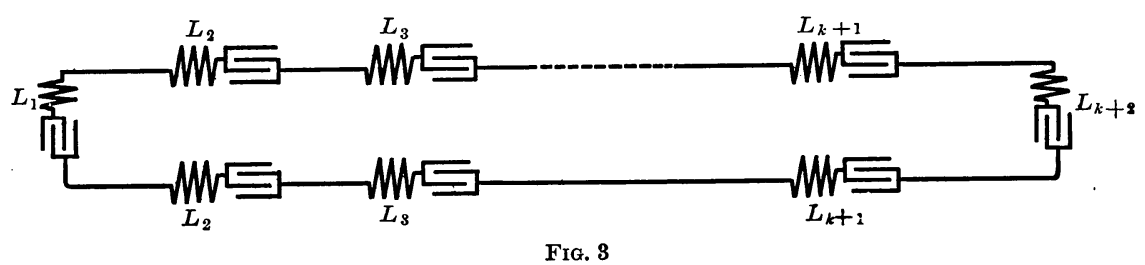

coils $L_{1}, L_{2}, \ldots$ To make the discussion more general, let each coil have a condenser of capacity $C_{0}$ in series with it.

Let $L, R$, and $C$, represent the inductance, resistance and capacity, respectively, per unit length of the uniform wire. Let an e. m. f. of type $E e^{i p t}$ be impressed at $L_{1}$. Employing the usual notation we shall have the following equation of propagation at every point of the uniform wire:

$$
L \frac{d^{2} y}{d t^{2}}+R \frac{d y}{d t}=\frac{1 \partial^{2} y}{C \partial s^{2}}
$$

the same as in $\S I$, but here the integral has to satisfy $2 k+2$ boundary conditions, one at each coil. On account of the symmetry of distribution of the coils on the two sides of the loop this number is reduced to $k+2$. 
To formulate these boundary conditions introduce the following notation :

Let $2 l$ be the length of the loop ; $l /(k+1)$ will be the distance between two consecutive coils. Let $L_{0}, R_{0}$, and $C_{0}$, be the inductance, resistance, and capacity, respectively, of each coil. Let $y_{m}$ be the current at any point of the interval between the coils $L_{m}$ and $L_{m+1}$. Denote the distance of this point from coil $L_{m}$ by $\xi$.

The currents in the coils will be denoted by $x_{1}, x_{2}, \cdots, x_{k+1}, x_{k+2}$. It is evident that

$$
\left(y_{m}\right)_{\xi=0}=\left(y_{m-1}\right)_{\xi=l l k+1)}=x_{m} \text {. }
$$

The boundary conditions for all coils except $L_{1}$ and $L_{k+2}$ can now be stated. They are :

where

$$
\left(\frac{\partial y_{m-1}}{\partial \xi}\right)_{\xi=l /(k+1)}-\left(\frac{\partial y_{m}}{\partial \xi}\right)_{\xi=0}=-C\left(-p^{2} \lambda_{0}+i p R_{0}\right) x_{m}=-h x_{m}
$$

$$
\lambda_{0}=L_{0}-\frac{1}{p^{2} C_{0}},
$$

There are $k$ equations of this form. The boundary equations for the first and the last coil are of the following forms:

for the first coil, and

$$
-2\left(\frac{\partial y_{1}}{\partial \xi}\right)_{\xi=0}=i p C E e^{i p t}-h x_{1}=D-h x_{1}
$$

for the last coil.

$$
2\left(\begin{array}{c}
\partial y_{k+1} \\
\partial \xi
\end{array}\right)_{\xi=l /(k+1)}=-h x_{k+2}
$$

Equation (3) of $\S \mathrm{I}$ and equation $(6 a)$ of $\S$ II suggest the following trial solution :

$$
y_{m}=K_{1} \cos \mu \xi+K_{2} \sin \mu \xi,
$$

where $\mu$ has the same value as in $\S \mathrm{I}$.

When $\xi=0, y_{m}$ becomes $x_{m}$, and when $\xi=l /(k+1), y_{m}$ becomes $x_{m+1}$. Hence

Therefore

$$
x_{m}=K_{1}, \quad x_{m+1}=K_{1} \cos \frac{\mu l}{k+1}+K_{2} \sin \frac{\mu l}{k+1} .
$$

$$
y_{m}=\frac{x_{m} \sin \mu\left(\frac{l}{k+1}-\xi\right)+x_{m+1} \sin \mu \xi}{\sin \frac{\mu l}{k+1}} .
$$

We have now to determine the $k+2$ constants $x_{1}, x_{2}, \ldots, x_{k+2}$ from the $k+2$ boundary equations. 
Begin with the boundary equation at the coil $L_{1}$ :

Therefore

$$
\frac{\partial y_{m}}{\partial \xi}=\frac{\mu\left\{-x_{m} \cos \mu\left(\frac{l}{k+1}-\xi\right)+x_{m+1} \cos \mu \xi\right\}}{\sin \frac{\mu l}{k+1}} .
$$

Let

$$
-2\left(\frac{d y_{1}}{d \xi}\right)_{\xi=0}=2\left(x_{1} \cos \frac{\mu l}{k+1}-x_{2}\right) \frac{\mu}{\sin \frac{\mu l}{k+1}}=D-h x_{1} .
$$

$$
\sigma=h \frac{\sin \frac{\mu l}{k+1}}{\mu}-4 \sin ^{2} \frac{1}{2} \frac{\mu l}{k+1}, \quad \rho=\frac{\sin \frac{\mu l}{k+1}}{\mu} D
$$

the boundary equations can then be written :

$$
\left\{\begin{array}{l}
(\sigma+1) x_{1}-0-x_{2}=\frac{\rho}{2}+\frac{\sigma}{2} x_{1}, \\
(\sigma+2) x_{2}-x_{1}-x_{3}=0 \\
(\sigma+2) x_{3}-x_{2}-x_{4}=0 \\
\cdot \cdot \cdot \cdot \cdot \\
(\sigma+2) x_{m}-x_{m-1}-x_{m+1}=0 \\
\cdot \cdot \cdot \cdot \cdot \cdot \\
(\sigma+2) x_{k+1}-x_{k}-x_{k+2}=0 \\
(\sigma+1) x_{k+2}-x_{k+1}-0=\frac{\sigma}{2} x_{k+2}
\end{array}\right.
$$

This system is of the same form as system (6) of $\S$ II. Equation (7) of that section enables us to write down the solution of (3).

Let

$$
\sigma+2=2 \cos 2 \psi
$$

then $x_{m}, y_{m}$ have the respective values:

$$
\left.-\frac{\left[\cos 2(k-m+2) \psi \sin \mu\left(\frac{l}{k+1}-\xi\right)+\cos 2(k-m+1) \psi \sin \mu \xi\right.}{k \sin 2 \psi \sin 2(k+1) \psi}\right]_{-} D
$$


These statements give the complete mathematical representation of waves of forced period on a conductor of this kind. The angles $\psi$ and $\mu$ are, of course, complex; hence, in order to calculate from these equations in any particular case the principal quantities with which we are concerned in wave propagation, namely, the wave-length and the attenuation constant, it would be necessary to study separately the real and the imaginary parts of these equations. This study in its broadest aspect presents considerable mathematical complexity. It is, however, somewhat remote from the principal aim of this paper which is to ascertain the conditions under which a non-uniform conductor of the second type is approximately equivalent to its corresponding uniform conductor, that is, to ascertain the frequencies for which the two will have approximately the same wave-lengths and the same attenuation constants.

\section{Equivalence of a non-uniform conductor of the second type to its corresponding uniform conductor.}

The solution of this problem is obtained by studying the following equation :

or

$$
\frac{h_{s} \sin \frac{\mu_{s} l}{k+1}}{\mu_{s}}+2 \cos \frac{\mu_{s} l}{k+1}=2 \cos 2 \psi_{s},
$$

$$
\begin{gathered}
\mu_{s} \sin k+1 \\
\mu_{s}
\end{gathered}
$$

The subscript $s$ denotes that the particular frequency speed $p_{s}$ is considered, and

$$
\mu_{s}=a_{s}+i \beta_{s}, \quad a_{s} \geqq \beta_{s} .
$$

If $\lambda_{s}$ is the wave-length corresponding to $p_{s}$ on the uniform wire before the introduction of the reactance points, then

$$
\frac{l}{k+1} a_{s}=\frac{l}{k+1} \lambda_{s} \text {. }
$$

Let $\theta_{s}$ be the angular distance between two consecutive coils for wave-length $\lambda_{s}$; then

therefore

$$
2 \pi: \lambda_{s}:: \theta_{s}: \frac{l}{k+1}
$$

$$
\theta_{s}=\frac{l}{k+1} \frac{2 \pi}{\lambda_{s}}=\frac{l}{k+1} a_{s} .
$$

Trans. Am. Math. Soc. 19 
Hence if $\theta_{s}$ is so small that $\sin \theta_{s}=\theta_{s}$ very nearly, then

or

$$
\frac{l}{k+1} h_{s}-\left(\frac{l}{k+1}\right)^{2} \mu_{s}^{2}=-4 \sin ^{2} \psi_{s},
$$

$$
\left(\frac{l}{k+1}\right)^{2} C\left\{-p_{s}^{2}(\rho+L)+i p(r+R)\right\}=-4 \sin ^{2} \psi_{s}
$$

where $\rho$ and $r$ are the inductance and resistance, respectively, per unit length of the inserted coils, so that $\rho+L$ and $r+R$, are the inductance and resistance respectively, of the corresponding uniform conductor. This last equation can also be written :

$$
\frac{l}{2} \frac{l}{k+1}\left(a_{s}^{\prime}+i \beta_{s}^{\prime}\right)=\sin \psi_{s} .
$$

In this case also $a_{s}^{\prime} \geqq \beta_{s}^{\prime}$.

Let $\lambda_{s}^{\prime}$ be the wave-length for frequency speed $p_{s}$ on the corresponding uniform conductor. Then

$$
a_{s}^{\prime}=\frac{2 \pi}{\lambda_{s}^{\prime}}
$$

Put $\phi_{s}^{\prime}=l a_{s}^{\prime} /(k+1)$; then $\phi_{s}^{\prime}$ is the angular distance between two consecutive coils, the angular distance of $\lambda_{s}^{\prime}$ being $2 \pi$.

If, therefore, $\frac{1}{2} \phi_{s}^{\prime}$ is so small that $\sin \frac{1}{2} \phi^{\prime}=\frac{1}{2} \phi^{\prime}$ very nearly, then

very nearly.

$$
\psi_{s}=\frac{1}{2} \frac{l}{k+1}\left(a_{s}^{\prime}+i \beta_{s}^{\prime}\right)=\frac{1}{2} \frac{l}{k+1} \mu_{s}^{\prime},
$$

Making this substitution in (4) we obtain equation (5a) of $\S \mathrm{I}$. The equation of wave propagation for a non-uniform conductor of the second type is, under these conditions, the same as that for its corresponding uniform conductor. The two will have approximately the same wave-length and the same attenuation constant for the frequency speed $p_{s}$.

The degree of approximation is the same as that of $\sin \psi_{s} / 2$ to $\psi_{s} / 2$.

A numerical example will illustrate this point more clearly.

Consider a uniform telegraph wire having the following constants per mile :

$$
\begin{aligned}
& L=.004 \text { Henry }, \\
& R=7 \text { Ohm }, \\
& C=.01 \times 10^{-6} \text { Farads } .
\end{aligned}
$$

Introduce at each mile a coil having

$$
\begin{aligned}
& L_{1}=.036 \text { Henry }, \\
& R=1 \text { Ohm } .
\end{aligned}
$$


The non-uniform conductor thus obtained will have for its corresponding uniform conductor a uniform wire having, per mile,

$$
\begin{aligned}
L+L_{1} & =.04 \text { Henry } \\
R+R_{1} & =8 \text { Ohm }, \\
C & =.01 \times 10^{-6} \text { Farads } .
\end{aligned}
$$

For a frequency of 1500 p. p. s. we shall have $p_{s}=2 \pi \times 1500$.

The wave-length $\lambda_{*}^{\prime}$ on the corresponding uniform conductor for this frequency is very nearly

$$
\lambda_{s}^{\prime}=33 \text { miles. }
$$

Hence, since the interposed coils are one mile apart, we shall have

$$
\sin \frac{1}{2} \phi^{\prime}=\sin \frac{\pi}{3 \overline{3}} \text {. }
$$

Now $\sin \pi / 33=\pi / 33$ to within $\frac{1}{6}$ of one per cent of the value of $\pi / 33$. Up to this degree of approximation the non-uniform conductor just described will have the same wave-length and the same attenuation constant as its corresponding uniform conductor. For lower frequencies the degree of approximation will be much higher; hence for all frequencies which are of any importance in the telephonic transmission of speech the two conductors are equivalent.

\section{B. Waves of free period.}

Equation (4) holds for all values of $D$; it should therefore hold good when $D=0$. In this case, however, all the currents will vanish unless the denominator vanishes, that is, unless

$$
\sin 2 \psi \sin 2(k+1) \psi=0 .
$$

This transcendental equation together with the equation :

$$
\frac{h}{\mu} \sin \frac{\mu l}{k+1}+2 \cos \frac{\mu l}{k+1}=2 \cos 2 \psi,
$$

determines the free periods and their corresponding damping constants.

Equation (4a) will be satisfied when

$$
\psi=\frac{r \pi}{2(k+1)},
$$

where $r$ is any integer from 0 to $\infty$.

When $r=s(k+1)$ we shall have not only $\sin 2(k+1) \psi=0$, but also $\sin 2 \psi=0$. 
These particular values of $\psi$ give a set of free oscillations which form a distinct group, called further below the oscillations of normal period.

Equation (4b) should therefore be written :

$$
\frac{h_{r} \sin \frac{\mu_{r} l}{k+1}}{\mu_{r}}+2 \cos \frac{\mu_{r} l}{k+1}=2 \cos \frac{r \pi}{k+1} .
$$

This equation will be referred to as the period equation.

It should be observed that

$$
-\mu_{r}^{2}=C\left(\nu_{r}^{2} L+\nu_{r} R\right), \quad h_{r}=C\left(\nu_{r}^{2} \lambda_{0}+\nu_{r} R_{0}\right) .
$$

An inspection of (5) shows that the complete solution for waves of free period can now be written:

$$
\begin{aligned}
& y_{m}=\sum_{r=1}^{\infty} \frac{A_{r}}{\sin \frac{r \pi}{k+1}}\left\{\cos (k-m+2) \frac{r \pi}{k+1} \sin \mu_{r}\left(\frac{l}{k+1}-\xi\right)\right. \\
& \left.+\cos (k-m+1) \frac{r \pi}{k+1} \sin \mu_{r} \xi\right\}, \\
& y_{k}=\sum_{r=1}^{\infty} \eta_{r}
\end{aligned}
$$

In the case of a uniform wire the periods and the wave-lengths of the simple harmonic components. $\eta_{1}, \eta_{2}, \ldots, \eta_{r}, \ldots$ have in general a harmonic relation. The introduction of the reactance points $L_{1}, L_{2}, \ldots$ disturbs this relation in consequence of a displacement in the values of the normal periods and of the normal damping constant. It is evident from purely physical considerations that since the introduction of the reactance points increases, in general, the inductance per unit length, the periods will be lengthened.

a. Waves of normal period.

According to equation (7), $\S$ I the wave-lengths of free oscillations on a uniform loop are as follows :

$$
\begin{array}{r}
\frac{2 l}{1}, \frac{2 l}{2}, \frac{2 l}{3}, \cdots, \frac{2 l}{k+1}, \frac{2 l}{k+2}, \cdots, \frac{2 l}{2(k+1)}, \frac{2 l}{2(k+3)}, \\
\cdots, \frac{2 l}{s(k+1)}, \frac{2 l}{s(k+1)+1}, \cdots .
\end{array}
$$

Divide this series into groups of $(k+1)$ members each. Consider now the last member of each group; call it the terminal member. The wave-lengths of the terminal members are 


$$
\frac{2 l}{(k+1)}, \frac{2 l}{2(k+1)}, \cdots, \frac{2 l}{s(k+1)}, \cdots
$$

These terminal members will appear with normal periods among the free oscillations on the periodically loaded loop. To show this consider the components of $y_{m}$ of type $\eta_{s(k+1)}$ where $s$ may be any integer between 1 and $\infty$. Since in the case of these components $\psi=s \pi$, the period equation becomes :

$$
\frac{h}{\mu} \sin \frac{\mu l}{k+1}+2 \cos \frac{\mu l}{k+1}=2 \cos s \pi .
$$

This equation will be satisfied by putting

$$
\mu=\frac{(k+1) s \pi}{l}
$$

The free periods of components of type $\eta_{s(k+1)}$ are now easily obtained :

$$
\nu_{s}^{2} L C+\nu_{s} R C=-\mu^{2}=-\frac{(k+1)^{2} s^{2} \pi^{2}}{l^{2}} .
$$

Therefore

$$
\nu_{s}=-\frac{R}{2 L} \pm i \sqrt{\frac{1}{L C} \frac{(k+1)^{2} s^{2} \pi^{2}}{l^{2}}-\frac{R^{2}}{4 L^{2}}}=-\frac{R}{2 L} \pm i p_{\bullet} .
$$

From the expression for $y_{m}$ we deduce:

where

$$
\eta_{s(k+1)}=B_{s(k+1)} \sin \frac{(k+1) s \pi \xi}{l},
$$

$$
B_{s(k+1)}=B_{s(k+1)}^{\prime} e^{-\frac{R}{2 L}} \cos \left(p_{s} t-\epsilon_{s}\right) .
$$

The wave-length of this component is $2 l / s(k+1)$.

The wave-lengths of the component of type $\eta_{s(k+1)}$ are

$$
\frac{2 l}{k+1}, \frac{2 l}{2(k+1)}, \frac{2 l}{3(k+1)}, \cdots, \frac{2 l}{s(k+1)}, \cdots
$$

From the wave-lengths and their corresponding periods we see that the oscillations of the type $\eta_{s(k+1)}$ are identical with the terminal members of the groups into which the free oscillations on a uniform loop were divided above. The physical reason why these periods have not been disturbed by the introduction of reactance points is easily seen; it is because they have their nodes at the reactance points. The other $k$ members'of each group have been disturbed by the presence of the reactance points. The character of this disturbance will now be discussed. 
b. Waves of displaced period.

From the expression for $y_{m}$ we deduce, by putting $\xi=0$,

$$
\begin{aligned}
x_{m} & =\sum_{r=1}^{\infty} \frac{A_{r} \cos (k-m+2) \frac{r \pi}{k+1} \sin \mu_{r} k+1}{\sin \frac{r \pi}{k+1}} \\
& =\sum_{r=1}^{\infty} \xi_{m, r} .
\end{aligned}
$$

First, consider the wave-lengths of these oscillations of type $\xi_{m, r}$. Begin with the fundamental :

$$
\xi_{m, 1}=\frac{A_{1} \cos (k-m+2) \frac{\pi}{k+1} \sin \mu_{1} k+1}{\sin \frac{\pi}{k+1}} .
$$

As $m$ increases from 1 to $k+2$ the angle $(k-m+2) \pi /(k+1)$ diminishes from $\pi$ to 0 . In Fig. 4, the line $L_{1} L_{1}$ represents the length of the loop, $L_{k+2}$ is the middle point of it. Points $1,2,3, \ldots, k+2$ mark the position of the

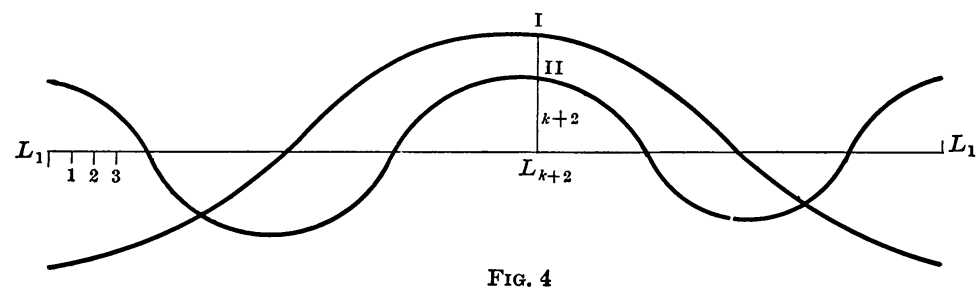

reactance points. At these points measure off abscissæ equal to $\xi_{1,1} \xi_{2,1} \xi_{3,1} \ldots$ The extremities of these abscissæ will be on the harmonic curve I. This curve represents the fundamental oscillation of displaced period. Its wave-length is $2 l$, the same as the wave-length of the fundamental oscillation before the introduction of the reactance points.

The next component is

$$
\xi_{m, 2}=\frac{A_{2} \cos (k-m+2) \frac{2 \pi}{k+1} \sin \mu_{2} \frac{l}{k+1}}{\sin \frac{2 \pi}{k+1}} .
$$

As $m$ increases from 1 to $k+2$ the angle $(k-m+2) 2 \pi /(k+1)$ diminishes from $2 \pi$ to 0 . It will therefore have four maxima and four zero points on the loop. Its wave-length is $l$. This component is represented by curve II in Fig. 4 . 
Take now the last component but one of the first group of free oscillations of displaced period. It is

$$
\xi_{m, k}=\frac{A_{k} \cos (k-m+2) \frac{k \pi}{k+1} \sin \frac{\mu_{k} l}{k+1}}{\sin k \pi+1} .
$$

As $m$ increases from 1 to $k+2$, the angle $(k-m+2) k \pi_{/}^{\prime}(k+1)$ diminishes from $k \pi$ to 0 . This component has therefore $2 k$ maxima and $2 k$ zero points on the loop. Its average wave-length is $2 l / k$. The last component of this group is the same as the lowest oscillation of normal period, that is, $\eta_{k+1}$.

The components of the first group have therefore the same average wave-lengths as the components of the first group of free oscillations on the loop before the introduction of the reactance points.

It can now be easily shown that this is true for the components of all the other groups and attention should be called here to the fact that the wave-lengths of the free oscillations of displaced periods can be considerably smaller than the distance between two consecutive reactance points. The introduction of the reactance points changes, therefore, neither the number of free oscillations nor their average wave-length. Their periods, however, and their damping constants will be changed as will be shown presently.

c. Displacement of periods.

The periods and damping constants of the oscillations of displaced periods are determined from the period equation:

$$
\frac{h_{r} \sin \frac{\mu_{r} l}{k+1}}{\mu_{r}}+2 \cos \frac{\mu_{r} l}{k+1}=2 \cos \frac{r \pi}{k+1} .
$$

The unknown quantity $\nu_{n}$, contained in $h_{r}$ and $\mu_{r}$, is, of course, a complex quantity, that is, we can write

$$
\nu_{r}=n_{r}+i p_{r}
$$

I am not as yet quite ready to discuss fully the general properties of the roots of the period equation. Besides, such a discussion would go considerably beyond the limits of this paper, the principal object of which is to determine the conditions under which the non-uniform conductor of Fig. 3 is equivalent to a uniform loop. I shall limit myself, therefore, to a brief statement of those properties, only, of this equation which bear directly upon these conditions.

First, since

$$
\cos \frac{r \pi}{k+1}=\cos \frac{[2 s(k+1) \pm r] \pi}{k+1},
$$


it follows that the periods and the damping constants of the wave-lengths

$$
\stackrel{2 l}{r}, \frac{2 l}{2(k+1) \pm r}, \frac{2 l}{4(k+1) \pm r}, \cdots, \frac{2 l}{2 s(k+1) \pm r}, \cdots,
$$

are determined from one and the same angle $\psi=r \pi /(k+1)$. This relation between these wave-lengths is illustrated graphically in Fig. 5. The horizontal top bar marked $1,2, \cdots, k+1, \cdots, 2 k+1,2 k+2,2 k+3, \cdots$ contains a numerical record of all the possible wave-lengths

$$
\stackrel{2 l}{1}, \frac{2 l}{2}, \cdots, \frac{2 l}{k+1}, \cdots, \frac{2 l}{2 k+1}, \cdots, \frac{2 l}{2 k+2}, \frac{2 l}{2 k+3}, \cdots
$$

The first vertical column marked $0,1,2,3, \ldots, k+1, \ldots$ contains the record of all possible values of $r$. The meaning of the black dots is now easily

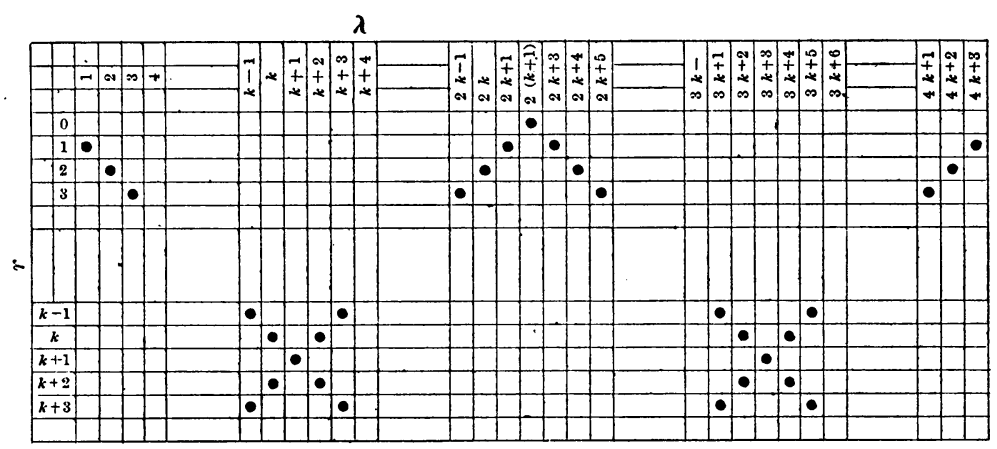

FIG. 5

explained. Take for instance the row of black dots in the horizontal bar 3 . These dots are in the columns $3,2 k-1,2 k+5,4 k+1,4 k+7, \cdots$. This means that if, in the period equation :

$$
\frac{h}{\mu} \sin \frac{\mu l}{k+1}+2 \cos \frac{\mu l}{k+1}=2 \cos \frac{r \pi}{k+1},
$$

we put $r=3$, then the roots of the equation will give us the periods and the damping constants for the wave-lengths

$$
\frac{2 l}{3}, \frac{2 l}{2 k-1}, \frac{2 l}{2 k+5}, \cdots
$$

For this reason these may be called the concomitant wave-lengths. An inspection of this table shows that the concomitant wave-lengths are symmetrically arranged with respect to the wave-lengths of normal periods 


$$
\frac{2 l}{k+1}, \frac{2 l}{2(k+1)}, \frac{2 l}{4(k+1)}, \cdots
$$

Of a given series of concomitant wave-lengths there is one in each of the groups mentioned above. The first one of these groups contains the longest wavelengths; it may be called the fundamental group. The wave-lengths of this group together with their concomitant wave-lengths give all the possible wavelengths. If, in the period equation, the values $0,1,2, \cdots, k+1$ are successively given to $r$, the roots of the resulting $k+2$ equations will give the periods and damping constants not only for the wave-lengths of the fundamental group but also their concomitants, that is, for all the wave-lengths.

It should be observed that the more nearly $\cos r \pi /(k+1)$ approaches the values +1 or -1 , the more closely will the concomitant wave-lengths approach the wave-lengths of normal period around which they are symmetrically grouped, and therefore, the more closely will their periods approach the period of the central wave-length. For instance, the wave-lengths

$$
\frac{2 l}{2 k+1}, \frac{2 l}{2 k+3}, \frac{2 l}{4 k+3}, \frac{2 l}{4 k+5}, \cdots
$$

are concomitants of the wave-lengths $2 l / 1$. Their periods are obtained from the period equation by putting $r=1$. This makes $\cos r \pi /(k+1)$ approach the value +1 and therefore, the periods of wave-lengths

$$
2 k+1 \text { and } \frac{2 l}{2 k+3}, \frac{2 l}{4 k+3} \text { and } \frac{2 l}{4 k+5}, \cdots
$$

will approach the periods of the wave-lengths

$$
2(l+1), \frac{2 l}{4(k+1)}, \cdots
$$

which have been calculated above. When, therefore, $k$ is large, the roots of the period equation :

$$
{ }_{\mu}^{h} \sin \frac{\mu l}{k+1}+2 \cos \frac{\mu l}{k+1}=2 \cos \frac{r \pi}{k+1},
$$

come out in pairs of nearly equal magnitude for all values of $r$ which are small in comparison to $k$. This peculiar property of the roots of the period equation suggests a striking resemblance between the oscillations of a periodically loaded conductor and the luminous vibrations of an incandescent gaseous substance which, in my opinion, deserves serious attention. I expect to discusis this matter more fully on some future occasion. 
d. Equivalence of a non-uniform conductor of the second type to its corresponding uniform conductor.

Suppose that we now increase the number of reactance points without increasing the total reactance and resistance introduced into the line. In the expression :

put

then

$$
h=C\left(\nu^{2} \rho+\nu R\right),
$$

$$
\rho=\frac{l \lambda^{\prime}}{k+1}, \quad R=\frac{l R^{\prime}}{k+1}
$$

$$
h=\frac{l}{k+1} C\left(\nu^{2} \lambda^{\prime}+\nu R^{\prime}\right)=\frac{l}{k+1} h^{\prime} .
$$

As the number of reactance points is increased, $\lambda^{\prime}$ and $R^{\prime}$ are to be kept constant; hence $\rho$ and $R$ must vary inversely as $k+1$. When $k$ becomes infinite, all the wave-lengths different from zero will be in the first group of Fig. 5 between the columns 1 and $k+1$. The other columns of this table lose their physical meaning. To show what becomes of the period equation when $k$ approaches the limit $\infty$, write the period equation in the following form :

$$
\frac{\frac{l}{k+1} h^{\prime} \sin \frac{\mu l}{k+1}}{\mu}-4 \sin ^{2} \frac{1}{2} \frac{l \mu}{k+1}=-4 \sin _{2}^{2} \frac{1}{k+1}{ }_{k \pi}^{r \pi}
$$

Since wave-lengths different from zero are to be considered, only finite values of $r$ need be considered. Hence when $k=\infty$ the period equation becomes :

or

$$
l^{2}\left(h^{\prime}-\mu^{2}\right)=-r^{2} \pi^{2},
$$

or

$$
C\left\{\nu^{2} \lambda^{\prime}+\nu R^{\prime}+\nu^{2} L+\nu R\right\}=-\frac{r^{2} \pi^{2}}{l^{2}}
$$

$$
C\left\{\nu^{2}\left(\lambda^{\prime}+L\right)+\nu\left(R^{\prime}+R\right)\right\}=-r^{2} \pi^{2}
$$

This equation is, as it ought to be, the period equation of a uniform conductor of inductance $\lambda^{\prime}+L$, resistance $R^{\prime}+R$, and capacity $C$ per unit length. This conductor is the so-called corresponding uniform conductor of the periodically loaded loop.

When $k$ is large but not infinite the same relation will exist approximately, and it is proposed to ascertain now the degree of this approximation.

We have seen that $l \mu_{t}^{\prime}(k+1)$ varies with $r$ as follows :

$$
\frac{l \mu}{k+1}=s \pi, \text { where } \quad r=s(k+1),
$$


$s$ being any integer from 0 to $\infty$. Thus when

$$
\begin{aligned}
& \frac{r \pi}{k+1} \quad \text { is } \quad 0, \pi, 2 \pi, 3 \pi, 4 \pi, \cdots, \\
& \frac{\mu l}{k+1} \text { will be } 0, \pi, 2 \pi, 3 \pi, 4 \pi, \cdots
\end{aligned}
$$

Since $\mu l /(k+1)$ varies continuously with $r$ it follows that when $r \pi(k+1)$ is small, $\mu l /(k+1)$ will also be small since both vanish simultaneously. It is well to introduce here the physical meaning of $r /(k+1)$. Let $\lambda_{r}$ be the wave-length of the wave for which $\psi=r \pi / 2(k+1)$; then $\lambda_{r}=2 l / r$, and therefore

$$
\frac{r \pi}{k+1}=\frac{2 \pi}{\lambda_{r}} \frac{l}{k+1}=2 \pi \frac{l_{1}}{\lambda_{r}}
$$

where $l_{1}$ is the distance between two consecutive coils. Let $2 \pi$ be the angular distance of $\lambda_{r}$, and $\theta_{r}$ the angular distance of $l_{1}$ corresponding to the period of $\lambda_{r}$; then

Therefore

$$
\frac{l_{1}}{\lambda_{r}}=\frac{\theta_{r}}{2 \pi} .
$$

$$
\frac{2 \pi}{k+1}=\theta_{r}
$$

Since for small values of $2 \pi /(k+1)$ the angle $\mu l /(k+1)$ is nearly equal to $r \pi /(k+1)$ it follows that within these limits $\mu l /(k+1)$ is approximately equal to the angle $\theta_{r}$. It follows therefore that wave-lengths for which $\theta_{r}$ is sufficiently small, the period equation :

$$
\frac{l}{k+1} \cdot \frac{h^{\prime}}{\mu} \sin \frac{\mu l}{k+1}-4 \sin ^{2} \frac{1}{2} \frac{l \mu}{k+1}=-4 \sin ^{2} \frac{1}{2} \frac{r \pi}{k+1},
$$

can be written :

or

$$
\left(\begin{array}{c}
l \\
k+1
\end{array}\right)^{2} h^{\prime}-\left(\frac{l}{k+1}\right)^{2} \mu^{2}=-\left(\begin{array}{c}
r \pi \\
k+1
\end{array}\right)^{2}
$$

$$
C\left\{\nu_{r}^{2}\left(\lambda^{\prime}+L\right)+\nu_{r}\left(R^{\prime}+R\right)\right\}=-\left(\frac{r \pi}{k+1}\right)^{2}
$$

that is, the periodically loaded conductor has approximately the same period, damping constant, and, of course, the same wave-length as its corresponding uniform conductor. The degree of approximation is of the same order as the degree of approximation between one half of the angular distance separating two consecutive coils and the sine of that distance. 
It should be observed that if the whole loop is divided into a number of equal parts and the consecutive parts are connected to each other by mutual induction, then the non-uniform conductor thus obtained will act in the same way as the non-uniform conductor of Fig. 3 of this section. Another arrangement which is equivalent to the two arrangements so far described is obtained by placing bridges at periodic intervals of the uniform loop, each bridge consisting of a coil of proper resistance and inductance, and the distance between the bridges being adjusted in accordance with the general rule formulated here. The mathematical analysis of these two arrangements does not differ essentially from the one given here and can be easily worked out.

A rule governing the degree of approximation between a non-uniform conductor and its corresponding uniform conductor has thus been established, and it has been proved that the same rule is applicable to both types of non-uniform conductors and also to both forced and free oscillations. The principal object of this investigation has, therefore, been accomplished.

Columbia University. 